\title{
Design Of An Optofluidic Device For The Measurement Of The Elastic Modu- lus Of Deformable Particles
}

\author{
Massimiliano M. Villone ${ }^{1, *}$, Janine K. Nunes ${ }^{2}$, Howard A. Stone ${ }^{2}$, and Pier Luca Maffettone \\ ${ }^{1}$ Department of Chemical, Materials, and Manufacturing Engineering, University of Naples Federico II \\ ${ }^{2}$ Department of Mechanical and Aerospace Engineering, Princeton University
}

\begin{abstract}
Suspensions carrying deformable inclusions are ubiquitous in nature and applications. Hence, highthroughput characterization of the mechanical properties of soft particles is of great interest. Recently, a non-invasive optofluidic technique has been developed for the measurement of the interfacial tension between two immiscible liquids [8]. We have adapted such technique to the case of soft solid beads, thus designing a non-invasive optofluidic device for the measurement of the mechanical properties of deformable particles from real-time optical imaging of their deformation.

The device consists of a cylindrical microfluidic channel with a cross-section reduction in which we make initially spherical soft beads flow suspended in a Newtonian carrier. By imaging the deformation of a particle in real time while it goes through the constriction, it is possible to get a measure of its elastic modulus through a theoretically derived-correlation. We provide both experimental and numerical validation of our device.
\end{abstract}

Suspensions carrying soft inclusions are frequent in nature and applications, e.g., biological cell suspensions. Their behavior is strongly influenced by the mechanical properties of the suspended particles, thus being able to measure these properties could be crucial to understand and control suspension flow. Moreover, biological cells can modify their deformability and functionality depending on their health state [1], so the measurement of their mechanical properties can be a powerful tool to discriminate whether they are healthy or not. In the last twenty years, several methods have appeared in the literature for the measurement of the mechanical properties of deformable particles, like atomic force microscopybased techniques (see, e.g., [2] and the references therein), micropipette aspiration [3], compression between parallel plates [4], osmotic compression [5], and capillary micromechanics [6]. However, all the aforementioned techniques are quite time-consuming. Some of them are also 'invasive' for the investigated samples and the measured values of the mechanical properties can be influenced by the contact with the probe of the measuring instrument. In the very recent years, microfluidics is turning out to be suitable tool for the measurement of the mechanical properties of biological cells [7]. About a decade ago, Hudson and co-workers have developed a continuous-flow non-invasive microfluidic technique providing almost realtime measurement of the interfacial tension between a liquid droplet and an immiscible suspending liquid [8]. Their method offers considerable advantages in terms of device fabrication and operation ease, low costs, and high throughput. We propose an adaptation of such a technique to the measurement of the elastic modulus of deformable particles and we validate it through numerical simulations and experiments on homogeneous synthetic particles with sizes and elasticities in the range of interest for biological cells [1].

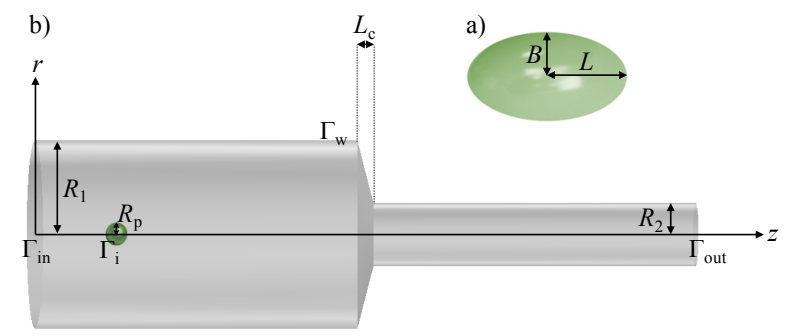

Figure 1. a) Schematic drawing of an elastic particle deformed into a prolate spheroid. b) Geometry of an initially spherical elastic bead suspended in a Newtonian liquid flowing through a device consisting of two coaxial cylindrical tubes.

In Fig. 1, the geometry of the device is depicted: an initially spherical elastic bead with radius $R_{\mathrm{p}}$ is suspended in a Newtonian liquid flowing through a cylindrical pipe with radius $R_{1}$. This channel undergoes an abrupt radial contraction to a narrow cylinder of radius $R_{2}$. While going through the constriction, the particle feels a time-dependent uniaxial extensional flow with extensional rate $\dot{\varepsilon}(t)$ that makes it deform into a prolate spheroid. If one monitors the transient response of the elastic bead to such flow and measures the deformation parameter $D(t)=$ $(L(t)-B(t)) /(L(t)+B(t))$ (see Fig. 1a) and its time derivative $d D / d t$, a plot of $\eta(15 / 8 \dot{\varepsilon}-d D / d t$ ) versus $D$ (with $\eta$

*e-mail: massimiliano.villone@unina.it 
the suspending liquid viscosity) should show a linear trend with a slope corresponding to the particle's elastic modulus $G$ [9]. Therefore, such a flow measurement provides a means for measuring $G$ in a continuous-flow process. This argument is conceptually analogous to that proposed by Hudson et al. for liquid droplets [8].
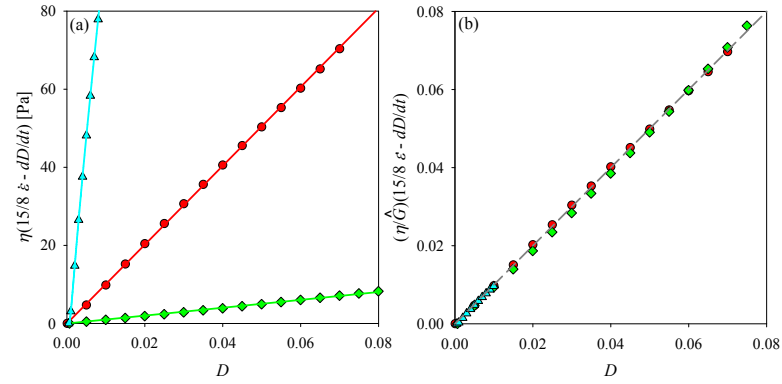

Figure 2. (a) Green diamonds, red circles, cyan triangles: Taylor plot of $\eta(15 / 8 \dot{\varepsilon}-d D / d t)$ versus $D$ for three different $G$-values. Green, red, cyan lines: linear fits of the three series of symbols (the slopes are equal to $100.71,1009.5,9998 \mathrm{~Pa}$, respectively). (b) Taylor plot of $(\eta / \hat{G})(15 / 8 \dot{\varepsilon}-d D / d t)$ versus $D$, with $\hat{G}$ the estimated value of the particle shear modulus, for the same data sets as in panel (a).

In order to validate our approach numerically, we have run simulations with beads having shear moduli of different orders of magnitude and verified that the aforementioned relation holds. Indeed, the data sets from numerical simulations appearing in Fig. 2a can be suitably fitted through linear functions with null intercept and a slope less than $1 \%$ different from the actual simulation input. In Fig. 2b, the sets of numerical results appearing in Fig. 2a are plotted each one scaled by the corresponding estimated value of the elastic modulus $\hat{G}$. As expected, the three se- ries of data all collapse on the bisector of the first quadrant of the Cartesian plane. Experiments on PEG-DA microgel particles provide a further proof of the validity of the proposed device.

\section{References}

[1] Fraldi, M and Cugno, A and Deseri, Luca and Dayal, $\mathrm{K}$ and Pugno, N M., Journal of the Royal Society Interface 12, 20150656 (2015)

[2] Tsukruk, Vladimir V and Singamaneni, Srikanth, Scanning Probe Microscopy of Soft Matter: Fundamentals and Practices, (John Wiley \& Sons, New York, 2012)

[3] Guilak, Farshid and Tedrow, John R and Burgkart, Rainer, Biochemical and Biophysical Research Communications 269, 781-786 (2000)

[4] Thoumine, Olivier and Ott, Albrecht and Cardoso, Olivier and Meister, Jean-Jacques, Journal of Biochemical and Biophysical Methods 39, 47-62 (1999)

[5] Saunders, Brian R and Vincent, Brian, Advances in Colloid and Interface Science 80, 1-25 (1999)

[6] Wyss, Hans M and Franke, Thomas and Mele, Elisa and Weitz, David A., Soft Matter 6, 4550-4555 (2010)

[7] Mietke, Alexander and Otto, Oliver and Girardo, Salvatore and Rosendahl, Philipp and Taubenberger, Anna and Golfier, Stefan and Ulbricht, Elke and Aland, Sebastian and Guck, Jochen and Fischer-Friedrich, Elisabeth, Biophysical Journal 109, 2023-2036 (2015)

[8] Hudson, Steven D and Cabral, Joao T and Goodrum Jr, William $\mathrm{J}$ and Beers, Kathryn L and Amis, Eric J, Applied Physics Letters 87, 081905 (2005)

[9] Roscoe, R, Journal of Fluid Mechanics 28, 273-293 (1967) 